\title{
Statistical Guarantee of QoS in Communication Networks with Temporally Correlated Loss
}

\author{
Homayoun Yousefi'zadeh Hamid Jafarkhani \\ Department of Electrical Engineering and Computer Science \\ University of California, Irvine \\ [hyousefi, hamidj] @uci.edu
}

\begin{abstract}
In this paper, we present an analytical study for the Gilbert loss model describing temporally correlated loss observed in the Internet and other communication systems. We obtain closed-form solutions describing transient and steady-state behavior of the model. Additionally starting from recursive equations of the Gilbert loss model, we derive closed-form solutions for arrival and loss patterns of the systems governed by the model. We show that utilizing our model yields to a lower complexity compare to the existing recursive models, attracting special attention of many different wired and wireless networking applications relying on such a model.
\end{abstract}

Index Terms-Temporally Correlated Loss, Burst Loss, Markov Chain, Gilbert Loss Model, Statistical Guarantee of QoS.

\section{INTRODUCTION}

$\mathbf{S}$ INCE the introduction of the so-called Gilbert model [4], it has been utilized to characterize the loss behavior of both wired and wireless communication systems. [2], [5], [6], and [10] are among many examples of the literature work utilizing the Gilbert loss model and its generalized multi-state models in wireless channels and networks. The model has also been in wide spread use in the literature of transport and error correcting computer communication systems. [1], [3], [7], [8], and [9] are among the examples of communication networks literature work suggesting utilization of the Gilbert loss model to capture temporally correlated loss in the Internet. The latter set of articles have all relied on the Gilbert model to introduce Forward Error Correction (FEC) techniques within the context of real-time media applications as well as reliable data transfer over multicast IP networks. Due to lack of an analytical model, these and many other literature articles have relied on employing dynamic programming techniques to solve recursive equations of the Gilbert loss model describing arrival and loss probabilities of the underlying system at the bit or packet level. In this article, we provide an analysis yielding to the introduction of closed-form solutions for the Gilbert loss model. Relying on the results of our analysis, we also propose a numerical algorithm with a significantly lower complexity than that of recursive methods to calculate arrival and loss probabilities of the system. Despite the fact that we refer to packet loss for the most part of this article, our results are nevertheless valid for bit loss as well.

An outline of the paper follows. In Section II, we obtain a closed-form solution for the time series representing state probabilities of the two-state Gilbert model along with the steadystate values of those time series. In Section III, we start from the describing recursive equations of the model to reach a closedform solution expressing arrival and loss patterns of systems governed by the model. In this section, we also provide an analysis of complexity for our resulting method and compare it with the existing recursive methods. In Section IV, we numerically verify that the results of our closed-form solution match the results of the recursive solution. Finally, we conclude the paper in Section V.

\section{The Two-State Gilbert Loss Model}

\section{A. General Description}

In this section, we briefly describe the two-state Gilbert loss model. As pointed out in [7], [9], and other articles, Internet and many other communication systems loss typically undergo burst loss representing temporally correlated loss. The Gilbert model provides an elegant mathematical model to capture the loss behavior of such systems. It is classified as an irreducible nonnull recurrent homogeneous discrete-time Markov chain. The transient and steady-state behavior of the Gilbert model can, hence, be studied using available time and frequency domain tools for such chains. In the Gilbert model, packet loss is described by a two-state model as illustrated in Fig. 1. The first state $G$ known as the GOOD state represents the receipt of a packet while the other state $B$ known as the BAD state represents the loss of a packet. While the GOOD state introduces a probability $P_{G}=\gamma$ of staying in the GOOD state and $1-P_{G}$ of transitioning to the BAD state, the BAD state introduces a probability $P_{B}=\beta$ of staying in the BAD state and $1-P_{B}$ of transitioning to the GOOD state.

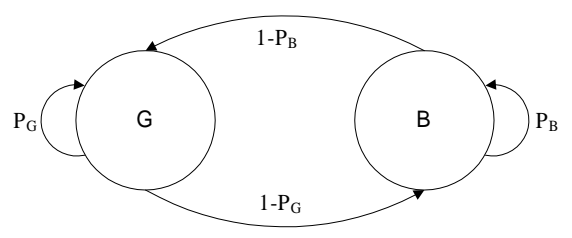

Fig. 1. The two-state Gilbert loss model with the state transition probabilities $1-P_{G}$ and $1-P_{B}$ for $P_{G}=\gamma$ and $P_{B}=\beta$.

\section{B. Analysis of the State Probabilities}

We start our analysis from the describing equations of the state probabilities of the Gilbert model. While the analysis of 
this section is standard [11], it is repeated for completeness. From Fig. 1, one can relate the probabilities of finding the system in the GOOD state $g[n+1]$ and the BAD state $b[n+1]$ at instant $n+1$ of the discrete time to their counter parts $g[n]$ and $b[n]$ at instant $n$. The describing difference equations are given by

$$
\begin{aligned}
& g[n+1]=\gamma g[n]+(1-\beta) b[n] \\
& b[n+1]=(1-\gamma) g[n]+\beta b[n]
\end{aligned}
$$

Next, we make the observation that at any instant of discrete time $n$, the property $g[n]+b[n]=u_{-1}[n]$ holds with $u_{-1}[n]$ indicating the unit step function. The latter observation is true due to the fact that at any instant of discrete time, the system is either in the GOOD or the BAD state implying the sum of two probabilities is one. Taking z-transform from both sides of the system of equations above, we carry the problem into the frequency domain as

$$
\begin{aligned}
\frac{1}{z}(G(z)-g[0]) & =\gamma G(z)+(1-\beta) B(z) \\
\frac{1}{z}(B(z)-b[0]) & =(1-\gamma) G(z)+\beta B(z)
\end{aligned}
$$

While $G(z)$ and $B(z)$ in Equation (2)denote the z-transforms of $g[n]$ and $b[n]$ series, $g[0]$ and $b[0]$ indicate the initial values of $g[n]$ and $b[n]$ series respectively. Solving the set of above equations relying on substitution and partial-fraction expansion, we obtain

$$
\begin{aligned}
G(z) & =\frac{((1-\beta)}{(2-\gamma-\beta)(1-z)}+\frac{(2-\gamma-\beta) g[0]-(1-\beta)}{(2-\gamma-\beta)(1-\gamma+\beta-1) z)} \\
B(z) & =\frac{((1-\gamma)}{(2-\gamma-\beta)(1-z)}+\frac{(2-\gamma-\beta) g[0]-(1-\beta)}{(2-\gamma-\beta)(1-(\gamma+\beta-1) z)}
\end{aligned}
$$

Extracting inverse z-transforms from equations of $G(z)$ and $B(z)$ yields the following time series for the probabilities of finding the system in the GOOD state and the BAD state in terms of state transition probabilities.

$$
\begin{aligned}
g[n] & =\frac{(1-\beta)}{(2-\gamma-\beta)} u_{-1}[n] \\
& +\frac{(2-\gamma-\beta) g[0]-(1-\beta)}{(2-\gamma-\beta)}(\gamma+\beta-1)^{n} u_{-1}[n] \\
b[n] & =\frac{(1-\gamma)}{(2-\gamma-\beta)} u_{-1}[n] \\
& +\frac{(2-\gamma-\beta) b[0]-(1-\gamma)}{(2-\gamma-\beta)}(\gamma+\beta-1)^{n} u_{-1}[n]
\end{aligned}
$$

We make note of the fact that $0 \leq \gamma+\beta \leq 2$ and hence $|\gamma+\beta-1| \leq 1$ implying convergence of the series. We also note that at instant $n=0$ the values of the series $g[n]$ and $b[n]$ from the above equations match their initial values $g[0]$ and $b[0]$ respectively.

At the end of this section, we note that steady-state values of the time series can be derived relying on the Final Value Theorem of the z-transform theory.

$$
\begin{aligned}
& g_{s s}=\lim _{z \rightarrow 1}(1-z) G(z)=\frac{1-\beta}{2-\gamma-\beta} \\
& b_{s s}=\lim _{z \rightarrow 1}(1-z) B(z)=\frac{1-\gamma}{2-\gamma-\beta}
\end{aligned}
$$

\section{Closed-Form Arrival And Loss Patterns}

While a review of the literature work reveals various recursive equations describing the behavior of the communication systems under the Gilbert loss model, to the best of our knowledge there is no study describing the loss behavior with closedform equations. Consistent with the proposed model of [9], it is observed from Fig. 1 that the recursive equations of the Gilbert loss model describing the probability of receiving $v$ packets from $u$ transmitted packets $P(u, v)$ are given by

$$
\begin{aligned}
P(u, v, G)= & P(u-1, v-1, G) \gamma \\
& +P(u-1, v-1, B)(1-\beta) \\
P(u, v, B)= & P(u-1, v, G)(1-\gamma)+P(u-1, v, B) \beta \\
P(u, v)= & P(u, v, G)+P(u, v, B)
\end{aligned}
$$

where $P(u, v, G)$ and $P(u, v, B)$ are the probabilities of receiving $v$ packets from $u$ transmitted packets and winding up in the GOOD state and the BAD state respectively. The describing initial conditions are given by

$$
\begin{aligned}
& P(v, 0, G)=0 \\
& P(v, v, B)=0 \\
& P(v, v, G)=\gamma^{v} g[0]+(1-\beta) \gamma^{(v-1)} b[0] \\
& P(v, 0, B)=(1-\gamma) \beta^{(v-1)} g[0]+\beta^{v} b[0]
\end{aligned}
$$

where $u, v \in\{1,2,3, \cdots\}, u \geq v$, and $g[0], b[0]$ are the probabilities that the system is initially in the GOOD state and the BAD state respectively.

Starting from the initial conditions and continuing on with the assumption that $u=v+z$, we use the set of iterative equations (6) to derive the following lemmas.

\section{Lemma 1:}

(a) The closed-form of equation $P(v+1, v, G)$ is given by

$$
\begin{aligned}
& P(v+1, v, G)=v \gamma^{v-1}(1-\beta)(1-\gamma) g[0] \\
& \quad+\gamma^{v-2}(1-\beta)[\beta \gamma+(v-1)(1-\beta)(1-\gamma)] b[0]
\end{aligned}
$$

(b) The closed-form of equation $P(v+1, v, B)$ is given by

$$
\begin{aligned}
& P(v+1, v, B)=\gamma^{v}(1-\gamma) g[0] \\
& \quad+\gamma^{v-1}(1-\beta)(1-\gamma) b[0]
\end{aligned}
$$

Lemma 2:

(a) The closed-form of equation $P(v+1, v-1, G)$ is given by

$$
\begin{aligned}
& P(v+1, v-1, G)= \\
& \gamma^{v-3}(1-\beta)(1-\gamma)\{ \\
& \sum_{i=0}^{1}\left(\begin{array}{c}
1 \\
i
\end{array}\right)\left(\begin{array}{c}
v-1 \\
i+1
\end{array}\right)(\beta \gamma)^{1-i}[(1-\beta)(1-\gamma)]^{i} \\
& \} g[0] \quad+\quad \gamma^{v-4}(1-\beta)\{ \\
& \sum_{i=0}^{2}\left(\begin{array}{c}
2 \\
i
\end{array}\right)\left(\begin{array}{c}
v-2 \\
i
\end{array}\right)(\beta \gamma)^{2-i}[(1-\beta)(1-\gamma)]^{i} \\
& \} b[0]
\end{aligned}
$$

(b) The closed-form of equation $P(v+1, v-1, B)$ is given by

$$
\begin{aligned}
& P(v+1, v-1, B)= \\
& \gamma^{v-2}(1-\gamma)\{ \\
& \sum_{i=0}^{1}\left(\begin{array}{c}
1 \\
i
\end{array}\right)\left(\begin{array}{c}
v-1 \\
i
\end{array}\right)(\beta \gamma)^{1-i}[(1-\beta)(1-\gamma)]^{i} \\
& \} g[0]+\gamma^{v-3}(1-\beta)(1-\gamma)\{ \\
& \sum_{i=0}^{1}\left(\begin{array}{c}
2 \\
i+1
\end{array}\right)\left(\begin{array}{c}
v-2 \\
i
\end{array}\right)(\beta \gamma)^{1-i}[(1-\beta)(1-\gamma)]^{i}
\end{aligned}
$$




\section{Lemma 3:}

(a) The closed-form of equation $P(v+2, v, G)$ is given by

$$
\begin{aligned}
& P(v+2, v, G)= \\
& \gamma^{v-2}(1-\beta)(1-\gamma)\{ \\
& \sum_{i=0}^{1}\left(\begin{array}{l}
1 \\
i
\end{array}\right)\left(\begin{array}{c}
v \\
i+1
\end{array}\right)(\beta \gamma)^{1-i}[(1-\beta)(1-\gamma)]^{i} \\
& \} g[0]+\gamma^{v-3}(1-\beta)\{ \\
& \quad \sum_{i=0}^{2}\left(\begin{array}{l}
2 \\
i
\end{array}\right)\left(\begin{array}{c}
v-1 \\
i
\end{array}\right)(\beta \gamma)^{2-i}[(1-\beta)(1-\gamma)]^{i} \\
& \quad\} b[0]
\end{aligned}
$$

(b) The closed-form of equation $P(v+2, v, B)$ is given by

$$
\begin{aligned}
& P(v+2, v, B)= \\
& \gamma^{v-1}(1-\gamma)\{ \\
& \sum_{i=0}^{1}\left(\begin{array}{c}
1 \\
i
\end{array}\right)\left(\begin{array}{c}
v \\
i
\end{array}\right)(\beta \gamma)^{1-i}[(1-\beta)(1-\gamma)]^{i} \\
& \} g[0] \quad+\gamma^{v-2}(1-\beta)(1-\gamma)\{ \\
& \quad \sum_{i=0}^{1}\left(\begin{array}{c}
2 \\
i+1
\end{array}\right)\left(\begin{array}{c}
v-1 \\
i
\end{array}\right)(\beta \gamma)^{1-i}[(1-\beta)(1-\gamma)]^{i} \\
& \} b[0]
\end{aligned}
$$

Proof: The proof of all of the lemmas is based on mathematical induction utilizing recursive equation set (6). In case of Lemma 1.(a), we verify that $P(v+1, v, G)$ holds assuming induction assumptions $P(v, v-1, G)$ and $P(v, v-1, B)$ hold. In case of Lemma 1. (b), we verify that $P(v+1, v, B)$ holds given the initial conditions $P(v, v, G)$ and $P(v, v, B)$. In case of Lemma 2.(a), we verify that $P(v+1, v-1, G)$ holds assuming induction assumptions $P(v, v-2, G)$ and $P(v, v-2, B)$ hold. In case of Lemma 2.(b), we verify that $P(v+1, v-1, B)$ holds given the results of Lemma 1.(a) and 1.(b). In case of Lemma 3.(a), we verify that $P(v+2, v, G)$ holds assuming induction assumptions $P(v+1, v-1, G)$ and $P(v+1, v-1, B)$ hold. In case of Lemma 3.(b), we verify that $P(v+2, v, B)$ holds given the results of the Lemma 2.(a) and 2.(b). The verification process is as follows.

Expanding summation terms of the right hand side of equation set (8), (9), (10), (11), (12), and (13) while relying on algebraic properties $\left(\begin{array}{l}j \\ i\end{array}\right)+\left(\begin{array}{c}j \\ i+1\end{array}\right)=\left(\begin{array}{l}j+1 \\ i+1\end{array}\right)$ and $\left(\begin{array}{l}j \\ 0\end{array}\right)=\left(\begin{array}{l}j \\ j\end{array}\right)=1$, we observe that the closed-form expressions for the right hand side of equation sets (8), (9), (10), (11), (12), and (13) are reduced to their left hand side counterparts. QED

We now generalize Lemma 3 for a fixed $z$ as

\section{Lemma $z+1$ :}

(a) The closed-form of equation $P(v+z, v, G)$ is given by

$$
\begin{aligned}
& P(v+z, v, G)= \\
& \gamma^{v-z}(1-\beta)(1-\gamma)\{ \\
& \sum_{i=0}^{z-1}\left(\begin{array}{c}
z-1 \\
i
\end{array}\right)\left(\begin{array}{c}
v \\
i+1
\end{array}\right)(\beta \gamma)^{z-1-i}[(1-\beta)(1-\gamma)]^{i} \\
& \} g[0]+\gamma^{v-z-1}(1-\beta)\{ \\
& \sum_{i=0}^{z}\left(\begin{array}{c}
z \\
i
\end{array}\right)\left(\begin{array}{c}
v-1 \\
i
\end{array}\right)(\beta \gamma)^{z-i}[(1-\beta)(1-\gamma)]^{i} \\
& \quad \begin{array}{l}
i \\
b[0]
\end{array}
\end{aligned}
$$

(b) The closed-form of equation $P(v+z, v, B)$ is given by

$$
\begin{aligned}
& P(v+z, v, B)= \\
& \gamma^{v-z+1}(1-\gamma)\{ \\
& \sum_{i=0}^{z-1}\left(\begin{array}{c}
z-1 \\
i
\end{array}\right)\left(\begin{array}{l}
v \\
i
\end{array}\right)(\beta \gamma)^{z-1-i}[(1-\beta)(1-\gamma)]^{i} \\
& \} g[0]+\gamma^{v-z}(1-\beta)(1-\gamma)\{ \\
& \sum_{i=0}^{z-1}\left(\begin{array}{c}
z \\
i+1
\end{array}\right)\left(\begin{array}{c}
v-1 \\
i
\end{array}\right)(\beta \gamma)^{z-1-i}[(1-\beta)(1-\gamma)]^{i} \\
& \} b[0] \quad z \geq 1, v \geq z
\end{aligned}
$$

Proof: The proof is based on mathematical induction. First, we verify that $P(v+z, v, G)$ satisfies the following equality assuming induction assumptions $P(v+z-1, v-1, G)$ and $P(v+z-1, v-1, B)$ hold.

$$
\begin{aligned}
& P(v+z, v, G)=\quad P(v+z-1, v-1, G) \gamma \\
& \quad+P(v+z-1, v-1, B)(1-\beta)
\end{aligned}
$$

Expanding summation terms of the right hand side of Equation (16) while relying on algebraic properties $\left(\begin{array}{l}j \\ i\end{array}\right)+\left(\begin{array}{c}j \\ i+1\end{array}\right)=\left(\begin{array}{l}j+1 \\ i+1\end{array}\right)$ and $\left(\begin{array}{l}j \\ 0\end{array}\right)=\left(\begin{array}{l}j \\ j\end{array}\right)=1$, we observe that the closed-form expressions for the right hand side of Equation (16) can be reduced to its left hand side as shown below.

$$
\begin{aligned}
& P(v+z-1, v-1, G) \gamma \\
& +P(v+z-1, v-1, B)(1-\beta)= \\
& \gamma^{v-z}(1-\beta)(1-\gamma)\{ \\
& \sum_{i=0}^{z-1}\left(\begin{array}{c}
z-1 \\
i
\end{array}\right)\left[\left(\begin{array}{c}
v-1 \\
i
\end{array}\right)+\left(\begin{array}{c}
v-1 \\
i+1
\end{array}\right)\right] \\
& (\beta \gamma)^{z-1-i}[(1-\beta)(1-\gamma)]^{i} \\
& \} g[0]+\gamma^{v-z-1}(1-\beta)\{ \\
& \left(\begin{array}{c}
z \\
0
\end{array}\right)\left(\begin{array}{c}
v-1 \\
0
\end{array}\right)(\beta \gamma)^{z} \\
& +\sum_{i=1}^{z}\left(\begin{array}{l}
z \\
i
\end{array}\right)\left[\left(\begin{array}{c}
v-2 \\
i-1
\end{array}\right)+\left(\begin{array}{c}
v-2 \\
i
\end{array}\right)\right] \\
& (\beta \gamma)^{z-i}[(1-\beta)(1-\gamma)]^{i} \\
& \} b[0] \\
& =P(v+z, v, G) \quad z \geq 1, v \geq z+2
\end{aligned}
$$

Next, we verify that $P(v+z, v, B)$ satisfies the following equality given the expressions of $P(v+z-1, v, G)$ and $P(v+z-1, v, B)$ from Lemma $z$.

$$
\begin{aligned}
& P(v+z, v, B)=\quad P(v+z-1, v, G)(1-\gamma) \\
& \quad+P(v+z-1, v, B) \beta
\end{aligned}
$$

Expanding summation terms of the right hand side of Equation (18) while relying on algebraic properties $\left(\begin{array}{l}j \\ i\end{array}\right)+\left(\begin{array}{c}j \\ i+1\end{array}\right)=\left(\begin{array}{l}j+1 \\ i+1\end{array}\right)$ and $\left(\begin{array}{l}j \\ 0\end{array}\right)=\left(\begin{array}{l}j \\ j\end{array}\right)=1$, we observe that the closed-form expressions for the right hand side of Equation (18) can be reduced to its left hand side as shown below.

$$
\begin{aligned}
& P(v+z-1, v, G)(1-\gamma) \\
& +P(v+z-1, v, B) \beta= \\
& \gamma^{v-z+1}(1-\gamma)\{ \\
& \left(\begin{array}{c}
z-1 \\
0
\end{array}\right)\left(\begin{array}{l}
v \\
0
\end{array}\right)(\beta \gamma)^{z-1} \\
& +\sum_{i=1}^{z-2}\left(\begin{array}{c}
v \\
i
\end{array}\right)\left[\left(\begin{array}{c}
z-2 \\
i-1
\end{array}\right)+\left(\begin{array}{c}
z-2 \\
i
\end{array}\right)\right] \\
& (\beta \gamma)^{z-1-i}[(1-\beta)(1-\gamma)]^{i} \\
& +\left(\begin{array}{c}
v \\
z-1
\end{array}\right)[(1-\beta)(1-\gamma)]^{z-1} \\
& \} g[0]+\gamma^{v-z}(1-\beta)(1-\gamma)\{ \\
& \sum_{i=0}^{z-2}\left(\begin{array}{c}
v-1 \\
i
\end{array}\right)\left[\left(\begin{array}{c}
z-1 \\
i
\end{array}\right)+\left(\begin{array}{c}
z-1 \\
i+1
\end{array}\right)\right] \\
& (\beta \gamma)^{z-1-i}[(1-\beta)(1-\gamma)]^{i} \\
& +\left(\begin{array}{l}
v-1 \\
z-1
\end{array}\right)\left(\begin{array}{c}
z-1 \\
z-1
\end{array}\right)[(1-\beta)(1-\gamma)]^{z-1} \\
& \} b[0] \\
& =P(v+z, v, B) \quad z \geq 2, v \geq z
\end{aligned}
$$


This concludes the proof. QED

Having proven Lemma $z+1$, we now state our main theorem.

\section{Theorem 1:}

For $\forall v, z \in\{1,2,3, \cdots\}$

(a) the closed-form expression for receiving exactly $v$ packets from $v+z$ transmitted packets and winding up in the GOOD state under the Gilbert loss model is given by Equation (14), and

(b) the closed-form expression for receiving exactly $v$ packets from $v+z$ transmitted packets and winding up in the BAD state under the Gilbert loss model is given by (15).

Proof: First we note that Theorem 1 is generalizing Lemma $z+1$ by claiming the accuracy of Equation (14) and Equation (15) for variables $v$ and $z$ rather than a variable $v$ and a fixed parameter $z$. The proof, hence, has to investigate two cases and is based on mathematical induction. In both cases the objective is to prove that Equation (16) and Equation (18) hold.

The first case considers the proof for a fixed $z$, showing that Equation (16) and Equation (18) hold for $v$ assuming they hold for $v-1$. We note that the entire proof of this case matches the proof of Lemma $z+1$.

The second case considers the proof for a fixed $v$, showing that Equation (16) and Equation (18) hold for $z$ assuming they hold for $z-1$. We note that the closed-form expression for $P(v+z-1, v-1, G)$ and $P(v+z-1, v-1, B)$ can be reached considering the proof of the first case above. The closed-form expression for $P(v+z-1, v-1, G)$ can be obtained as the result of replacing $v$ by $v-1$ in Equation (14). Likewise, the closed-form expression for $P(v+z-1, v-1, B)$ can be obtained as the result of replacing $v$ by $v-1$ in and Equation (15). We also note that the closed-form expression for $P(v+z-1, v, G)$ and $P(v+z-1, v, B)$ can be reached considering induction assumption. The closed-form expression for $P(v+z-1, v, G)$ can be obtained as the result of replacing $z$ by $z-1$ in Equation (14). Likewise, the closed-form expression for $P(v+z-1, v, B)$ can be obtained as the result of replacing $z$ by $z-1$ in Equation (15). Having explained the reasoning based on which the closed-form expressions of $P(v+z-1, v-1, G), P(v+z-1, v-1, B), P(v+z-1, v, G)$, and $P(v+z, v-1, B)$ can be extracted, reaching the left hand side of Equation (16) and Equation (18) from the right hand side counterparts is the same way described in Equation (17) and Equation (19) as a part of the proof of Lemma $z+1$.

QED

As an important consideration we can accurately replace the initial values of the time series $g[0]$ and $b[0]$ in Equation (14) and Equation (15) with their steady-state values $g_{s s}$ and $b_{s s}$ from Equation (5) assuming an arbitrarily far start instant of time for the Markov chain of Fig. 1.

We end this section by discussing the application of our results in error correcting systems. For a system undergoing burst loss, an error correcting system typically requires to rely on a method of calculating the smallest number of required trans- mitted packets $u$ in order to guarantee the receipt of at least $v$ packets with a probability $\Pi$ or better.

Assuming $u=v+z$, imposing a practical upper bound of $v$ on the value of $z$, and utilizing the fact that for an arbitrary probability model $P(u, v)$ indicating the probability of receiving exactly $v$ packets from $u$ transmitted packets, the probability of receiving at least $v$ packets from $u$ transmitted packets is given by $D(u, v)=\sum_{i=v}^{u} P(u, i)$, the following algebraic placement algorithm with a time complexity of $\mathcal{O}(v)$ can be used to accomplish the task.

\section{Statistical Guarantee for Packet Arrival Algorithm}

- for $(z=1$ to $v-1)\{$

- Calculate $P(v+z, v)=P(v+z, v, G)+P(v+$ $z, v, B)$ from Equation (14) and Equation (15).

- If $D(v+z, v) \geq \Pi \quad$ Break.

\} / for $(z=1$ to $v-1) * /$

- Report the number of required packets, $u=v+z$.

Comparing time complexity of the algorithm with that of a dynamic programming algorithm $\mathcal{O}\left(v^{2}\right)$ as proposed in [8], we observe that our algorithm is a much better performing approach.

Additionally, we make note of the fact that our proposed method is of special interest to error correcting systems relying on an apriori estimate of loss.

\section{NUMERICAL VERIFICATION}

In this section, we numerically verify that the results of the closed-form solutions of 14 and 15 for the number of arrived packets under the Gilbert model match the results of the recursive solutions of 16 and 18 . The simulation results span over two different ranges of parameter $v$. While Fig. 2 and Fig. 3 show our simulation results for the range $v \in[2,15]$, Fig. 4 and Fig. 5 show the results for the range $v \in[90,100]$. We note

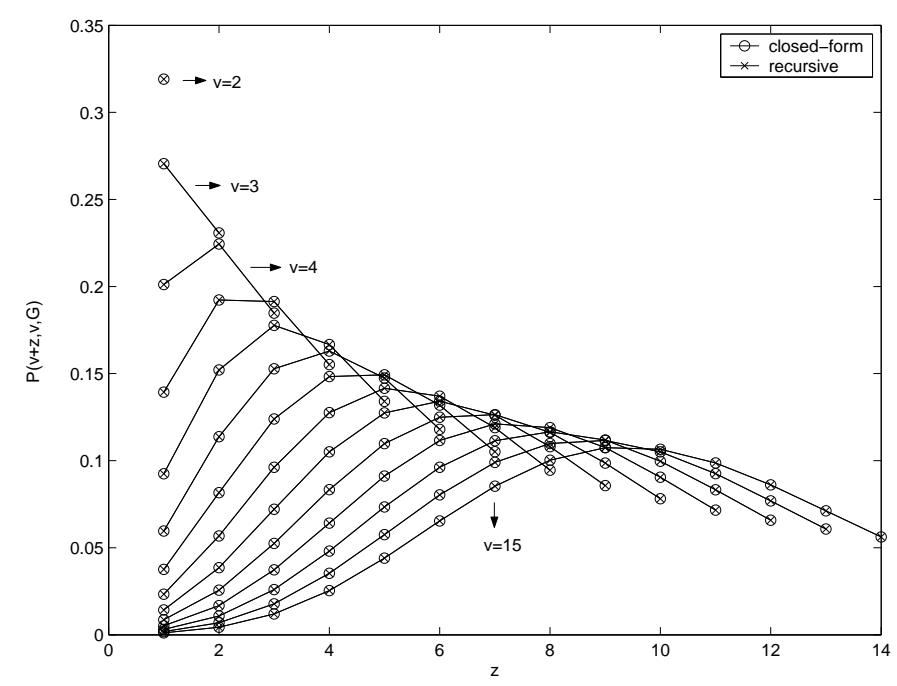

Fig. 2. A comparison plot of $P(v+z, v, G)$ vs $z$ between the packet arrival results of closed-form and iterative solutions for the choice of parameters $v \in$ $[2,15]$ and $z \in[1, v-1]$.

that the experiment results illustrated above are obtained for 


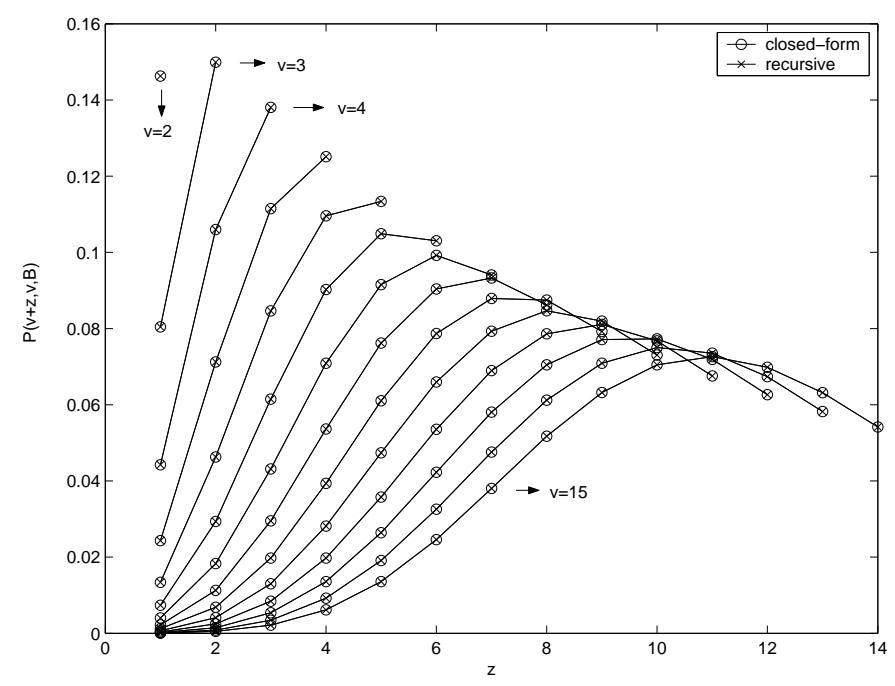

Fig. 3. A comparison plot of $P(v+z, v, B)$ vs $z$ between the packet arrival results of closed-form and iterative solutions for the choice of parameters $v \in$ $[2,15]$ and $z \in[1, v-1]$.

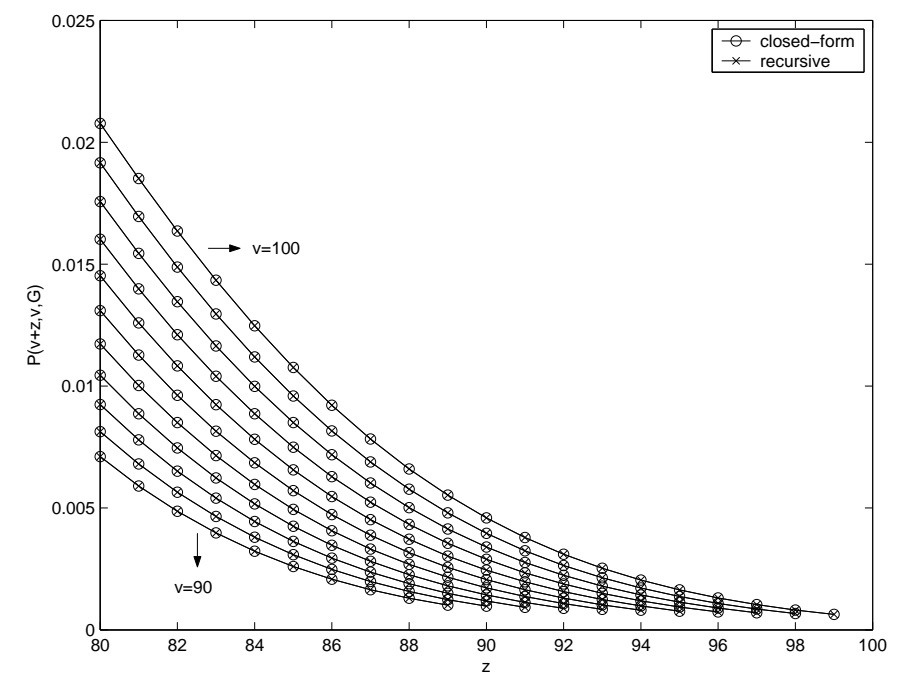

Fig. 4. A comparison plot of $P(v+z, v, G)$ vs $z$ between the packet arrival results of closed-form and iterative solutions for the choice of parameters $v \in$ $[90,100]$ and $z \in[80, v-1]$.

the choice of parameters $\gamma=0.55, \beta=0.35, g[0]=0.59$, and $b[0]=0.41$. As observed from the figures, the packet arrival results of closed-form and iterative solutions exactly match each other verifying the accuracy of our closed-form solution results.

\section{CONCLUSion AND Future Work}

In this paper, we obtained a closed-form solution for the time series representing state probabilities of the two-state Gilbert model along with the steady-state values of those time series. In addition, we obtained closed-form solutions describing packet arrival and loss patterns of the systems governed by the Gilbert loss model. Finally, we integrated our results within the context of error correcting communication systems and numerically verified the accuracy of our results.

We are currently working on generalizing our approach for multi-state Markov chains and anticipate introducing closedform solutions for such chains as the result of our future work.

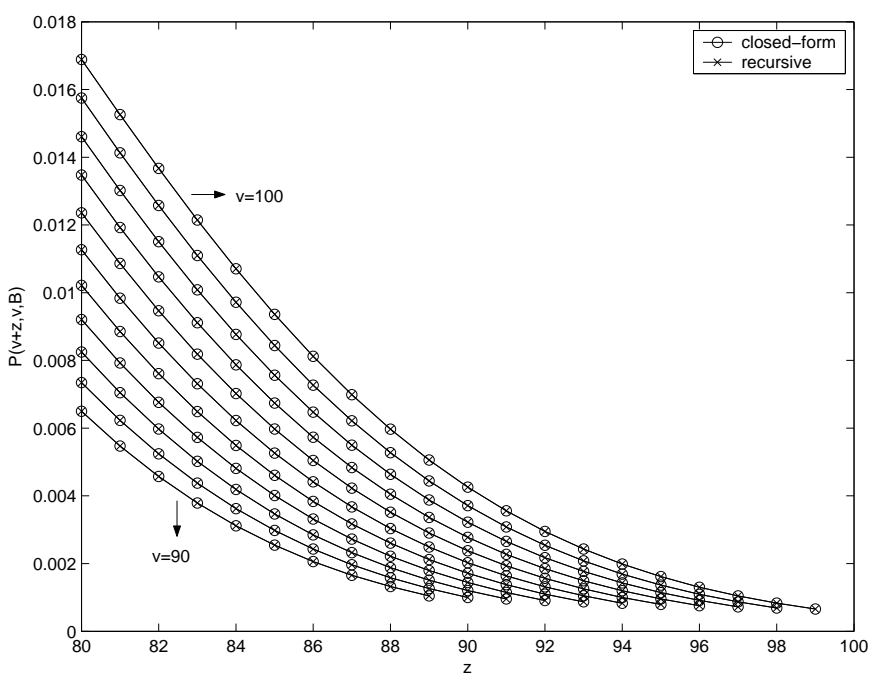

Fig. 5. A comparison plot of $P(v+z, v, B)$ vs $z$ between the packet arrival results of closed-form and iterative solutions for the choice of parameters $v \in$ $[90,100]$ and $z \in[80, v-1]$.

\section{ACKNOWLEDGMENT}

The authors would like to thank Yun Zhu for her assistance in preparing the simulation testbed.

\section{REFERENCES}

[1] E. Altman, K. Avrachenkov, and C. Barakat, "TCP in Presence of Bursty Losses," In Proc. of ACM SIGMETRICS, 2000.

[2] A. Balachandran, G. M. Voelker, P. Bahl, and P. V. Rangan, "Characterizing User Behavior and Network Performance in a Public Wireless LAN," In Proc. of ACM SIGEMTRICS, 2002.

[3] P. Frossard, O. Verscheure, "AMISP: A Complete Content-Based MPEG2 Error-Resilient Scheme," IEEE Trans. on Circuits and Syst. for Video Technology, September 2001.

[4] E.N. Gilbert, "Capacity of A Burst-Noise Channel," Bell Syst. Tech J., vol. 39, pp. 1253-1265, September 1960.

[5] H. Jafarkhani, P. Ligdas, N. Farvardin, "Adaptive Rate Allocation in a Joint Source/ Channel Coding Framework for Wireless Channels," In Proc. of IEEE VTC, April 1996.

[6] H. Liu, M. El Zarki, "Delay and Synchronization Control Middleware to Support Real-Time Multimedia Services over Wireless PCS Networks," IEEE JSAC, September 1999.

[7] J. Nonnenmacher, E. Biersack, D. Towsley, "ParityBased Loss Recovery for Reliable Multicast Transmission," In Proc. of ACM SIGCOMM, 1997.

[8] I. Rhee, S.R. Joshi, M. Lee, "Layered Multicast Recovery (LMR)," In Proc. of IEEE INFOCOM, 2000.

[9] D. Rubenstein, J. Kurose, D. Towsley, "Real-Time Reliable Multicast Using Proactive Forward Error Correction," In Proc. of NOSSDAV, 1998.

[10] H.S. Wang, N. Moayeri, "Finite-State Markov Channel: A Useful Model for Radio Communication Channels," IEEE Trans. on Vehicular Technology, February 1995.

[11] R. Yates, D.J. Goodman, "Probability and Stochastic Processes," Wiley, 1999. 